\title{
MATRICES ASSOCIATED WITH FRACTIONAL HANKEL AND FOURIER TRANSFORMATIONS
}

\author{
by A. P. GUINAND
}

(Received 20th August, 1955)

1. Introduction. Several writers (4), (6), (7), (9) have used orthogonal expansions in discussing properties of Fourier transformations, and Kober (3) has used such expansions to derive fractional Fourier and Hankel transformations. In 1950 Barrucand (1) noted a reciprocity holding between the coefficients in the expansions in Laguerre polynomials of pairs of functions which are transforms with respect to the kernel $J_{0}\left(2 x^{\frac{1}{2}}\right)$.

In the present paper I extend Barrucand's result to kernels $J_{\alpha}\left(2 x^{3}\right), R(\alpha)>-1$, and to Fourier sine and cosine kernels. I also discuss the relationship between fractional powers of unit matrices and fractional transformations, and I show how this method gives an alternative approach to the fractional Hankel transformations of Kober.

2. Formalities. Let us suppose that the sets of functions

$$
\left\{\phi_{n}(x)\right\}, \quad\left\{\psi_{n}(x)\right\} \quad(n=0,1,2, \ldots),
$$

are normalised and biorthogonal over $(0, \infty)$, that $f(x)$ and $g(x)$ are transforms with respect to a Fourier kernel $K(x)$, and that

and

$$
\begin{aligned}
& f(x) \sim \sum_{n=0}^{\infty} a_{n} \phi_{n}(x), \\
& g(x) \sim \sum_{n=0}^{\infty} b_{n} \phi_{n}(x)
\end{aligned}
$$

in the sense that* the coefficients $a_{n}$ and $b_{n}$ are given by

$$
a_{n}=\int_{0}^{\infty} f(x) \psi_{n}(x) d x, \quad b_{n}=\int_{0}^{\infty} g(x) \psi_{n}(x) d x .
$$

Further, suppose that $\Psi_{n}(x)$ is the transform of $\psi_{n}(x)$ with respect to the kernel $K(x)$ and that it can be expanded in terms of the functions $\psi_{n}(x)$. That is

$$
\Psi_{n}(x)=\int_{0}^{\infty} \psi_{n}(t) K(x t) d t=\sum_{m=0}^{\infty} k_{n, m} \psi_{m}(x)
$$

say. By the Parseval theorem for these transforms we have, formally,

$$
\begin{aligned}
& a_{n}=\int_{0}^{\infty} f(x) \psi_{n}(x) d x=\int_{0}^{\infty} g(x) \Psi_{n}(x) d x \\
& =\int_{0}^{\infty} g(x)\left\{\sum_{m=0}^{\infty} k_{n, m} \psi_{m}(x)\right\} d x \\
& =\sum_{m=0}^{\infty} k_{n, m} \int_{0}^{\infty} g(x) \psi_{m}(x) d x . \\
& =\sum_{m=0}^{\infty} k_{n, m} b_{m} .
\end{aligned}
$$

* The sign $\sim$ is used with this meaning throughout the paper. 
Simiarly

$$
b_{n}=\sum_{m=0}^{\infty} k_{n, m} a_{m}
$$

If $K, A, B$ denote the infinite matrix and the column vectors

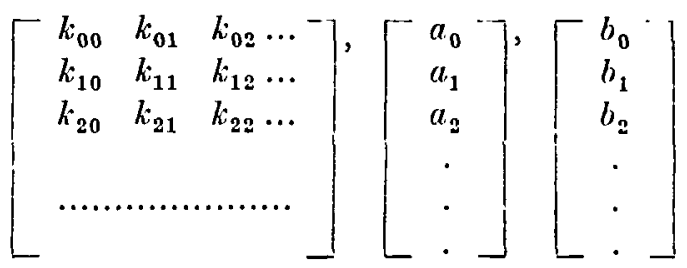

respectively, then (2) and (3) become $B=K A$ and $A=K B$. Hence $K^{2} A=A$ for all column vectors $A$ arising from the series (1), and thus $K^{2}=I$, where $I$ is the unit infinite matrix. Thus the Fourier kernel $K(x)$ may be regarded as corresponding to the square root $K$ of $I$.

In the same way other fractional powers of the unit infinite matrix $I$ correspond to formal transformations which are fractional powers of the transformation with respect to the Fourier kernel $K(x)$. In particular, the fractional transformations of Kober correspond to the diagonal matrix

$$
\left[\begin{array}{cccc}
1 & 0 & 0 & \cdots \\
0 & e^{2 \pi i k} & 0 & \ldots \\
0 & 0 & e^{4 \pi i k} & \cdots \\
\ldots \ldots \ldots \ldots \ldots \ldots \ldots \ldots \ldots \ldots
\end{array}\right]
$$

\section{Powers of Unit Matrices}

DeFINITION 1. If $I_{n}$ is the unit $n \times n$ matrix and $I(x)$ is an $n \times n$ matrix whose elements are functions of a parameter $x$ defined for all real $x$, then $I(x)$ is said to be an cvaluation of $I_{n}^{x}$, the xth power of $I_{n}$, if

$$
\begin{aligned}
& \text { (i) } I(x) \cdot I(y)=I(x+y) \text { for all real } x \text { and } y \text {, } \\
& \text { (ii) } I(0)=I(1)=I_{n} \text {. }
\end{aligned}
$$

With this terminology we have :

TheOREM 1. If $c_{1}, c_{2}, \ldots, c_{n-1}$ is any set of $n-1$ constants and $I(x)$ is the lower semi-matrix defined by

$$
l(x)=\left[\begin{array}{cccc}
a_{11} & a_{12} & \ldots & a_{1 n} \\
a_{21} & a_{22} & \ldots & a_{2 n} \\
\ldots \ldots \ldots \ldots \ldots \ldots \ldots \ldots \\
a_{n 1} & a_{n 2} & \ldots & a_{n n}
\end{array}\right],
$$

where $a_{r s}=0$ if $r<s$, and

$$
a_{r s}=\frac{1}{(r-s) !} e^{2 \pi i x(s-1)}\left(1-e^{2 \pi i x}\right)^{r-s} \prod_{m=8}^{r-1} c_{m}
$$

if $r \geqslant s$, then $I(x)$ is an evaluation of $I_{n}^{x}$. 
Proof. By the multiplication rule for matrices the element in the $r$ th row and sth column of the product $I(x) . I(y)$ is

$$
\begin{aligned}
& \sum_{p=8}^{r} \frac{e^{2 \pi i x(p-1)+2 \pi i y(s-1)}}{(r-p) !(p-s) !} c_{s} c_{s+1} \ldots c_{p-1} c_{p} c_{p+1} \ldots\left(1-e^{2 \pi i x}\right)^{r-p}\left(1-e^{2 \pi i y}\right)^{p-s} \\
& =\frac{1}{(r-s) !}\left\{\prod_{m=8}^{r-1} c_{m}\right\} e^{2 \pi i(x+v)(s-1)} \sum_{p=8}^{r}\left(\begin{array}{c}
r-s \\
r-p
\end{array}\right)\left(1-e^{2 \pi i x}\right)^{r-p}\left(e^{2 \pi i x}-e^{2 \pi i x+2 \pi i \nu}\right)^{p-s} \\
& =\frac{1}{(r-s) !}\left\{\begin{array}{l}
r-1 \\
\prod_{m=8}
\end{array} c_{m}\right\} e^{2 \pi i(x+y)(s-1)}\left\{1-e^{2 \pi i(x+y)}\right\}^{r-s}
\end{aligned}
$$

if $r \geqslant s$ and is zero if $r<s$. That is, condition (i) is satisfied. Condition (ii) follows immediately on substituting $x=0$ and $x=1$ in (5).

The result of Theorem 1 extends immediately to infinite lower semi-matrices, since the product of two lower semi-matrices is a lower semi-matrix and the elements of the rth row of the product depend only on the elements of the first $r$ rows of the two factor matrices. matrix

In particular, if we put $c_{m}=\frac{1}{2}(\alpha+m), x=\frac{1}{2}$, then it follows that the infinite lower semi-

$$
\left[\begin{array}{cccc}
\left(\begin{array}{c}
\alpha \\
0
\end{array}\right) & 0 & 0 & \ldots \\
\left(\begin{array}{c}
\alpha+1 \\
1
\end{array}\right) & -\left(\begin{array}{c}
\alpha+1 \\
0
\end{array}\right) & 0 & \ldots \\
\left(\begin{array}{c}
\alpha+2 \\
2
\end{array}\right) & -\left(\begin{array}{c}
\alpha+2 \\
1
\end{array}\right) & \left(\begin{array}{c}
\alpha+2 \\
0
\end{array}\right) & \ldots \\
\ldots \ldots \ldots \ldots \ldots \ldots \ldots \ldots \ldots \ldots \ldots \ldots \ldots \ldots \ldots \ldots \ldots \ldots \ldots \ldots \ldots
\end{array}\right]
$$

is a square root of the unit infinite matrix $I$.

Hence if $a_{0}, a_{1}, a_{2}, \ldots$ is any sequence of numbers and

then*

$$
\begin{aligned}
& b_{n}=\sum_{m=0}^{n}(-1)^{m}\left(\begin{array}{l}
n+\alpha \\
n-m
\end{array}\right) a_{m}, \\
& a_{n}=\sum_{m=0}^{n}(-1)^{m}\left(\begin{array}{l}
n+\alpha \\
n-m
\end{array}\right) b_{m} .
\end{aligned}
$$

4. Application to Hankel transforms. Suppose that $f(x)$ and $g(x)$ are transforms with respect to the kernel $\dagger J_{\alpha}(2 x), R(\alpha)>-1$, and that they have formal developments in Laguerre polynomials of the form

That is (see (5))

$$
\begin{aligned}
& f(x) \sim x^{1 \alpha} \sum_{n=0}^{\infty} a_{n} L_{n}^{(\alpha)}(x), \\
& g(x) \sim x^{1 \alpha} \sum_{n=0}^{\infty} b_{n} L_{n}^{(\alpha)}(x) .
\end{aligned}
$$

$$
\begin{aligned}
& a_{n}=\frac{n !}{\Gamma(n+\alpha+1)} \int_{0}^{\infty} e^{-x} x^{\ddagger \alpha} L_{n}^{(\alpha)}(x) f(x) d x, \\
& b_{n}=\frac{n !}{\Gamma(n+\alpha+1)} \int_{0}^{\infty} e^{-x} x^{\frac{1}{2} \alpha} L_{n}^{(\alpha)}(x) g(x) d x .
\end{aligned}
$$

- The case $\alpha=0$ gives the "reciprocal sequences" of Barrucand (1), corresponding to the Euler semi-matrix (2).

$\dagger \mathrm{In}$ this case $x^{\frac{1}{3}} f\left(\frac{1}{2} x^{2}\right)$ and $x^{\frac{3}{3}} g\left(\frac{1}{2} x^{2}\right)$ are transforms with respect to the usual Hankel kernel $x^{\frac{1}{2}} J_{\alpha}(x)$. 
Now the functions $n ! e^{-x} x^{\frac{1}{\alpha} \alpha} L_{n}^{(\alpha)}(x), e^{-x} x^{n+1 \alpha}$ are transforms $((5), 5 \cdot 4 \cdot 1)$ with respect to the kernel $J_{\alpha}\left(2 x^{2}\right)$. Hence, by the Parseval theorem for these transforms, (9) becomes

$$
a_{n}=\frac{1}{\Gamma(n+\alpha+1)} \int_{0}^{\infty} e^{-x} x^{n+1 \alpha} g(x) d x .
$$

Further $((5), 5 \cdot 1 \cdot 6)$

$$
L_{n}^{(\alpha)}(x)=\sum_{m=0}^{n}(-1)^{m}\left(\begin{array}{c}
n+\alpha \\
n-m
\end{array}\right) \frac{x^{m}}{m !} .
$$

Hence, by the reciprocity (7),

$$
\frac{x^{n}}{n !}=\sum_{m=0}^{n}(-1)^{m}\left(\begin{array}{c}
n+\alpha \\
n-m
\end{array}\right) L_{m}^{(\alpha)}(x)
$$

Substituting (11) in (10), we obtain

$$
\begin{aligned}
a_{n} & =\frac{n !}{\Gamma(n+\alpha+1)} \sum_{m=0}^{n}(-1)^{m}\left(\begin{array}{l}
n+\alpha \\
n-m
\end{array}\right) \int_{0}^{\infty} e^{-x} x^{\frac{1}{\alpha}} L_{m}^{(\alpha)}(x) g(x) d x \\
& =\frac{n !}{\Gamma(n+\alpha+1)} \sum_{m=0}^{n}(-1)^{m}\left(\begin{array}{c}
n+\alpha \\
n-m
\end{array}\right) \frac{\Gamma(m+\alpha+1)}{m !} b_{m} \\
& =\sum_{m=0}^{n}(-1)^{m}\left(\begin{array}{l}
n \\
m
\end{array}\right) b_{m} .
\end{aligned}
$$

Now it is not necessary for this argument that the series (8) should converge; the integrals (9) converge and the use of the Parseval theorem is justified if $f(x)$ belongs to $L^{2}(0, \infty)$. Thus we have :

THEOREM 2. If $f(x)$ belongs to $L^{2}(0, \infty)$ and $g(x)$ is its transform with respect to the kernel $J_{\alpha}\left(2 x^{\mathrm{t}}\right), R(\alpha)>-1$, and $a_{n}$ and $b_{n}$ are defined by $(9)$, then

and

$$
\begin{aligned}
& b_{n}=\sum_{m=0}^{n}(-1)^{m}\left(\begin{array}{l}
n \\
m
\end{array}\right) a_{m}, \\
& a_{n}=\sum_{m=0}^{n}(-1)^{m}\left(\begin{array}{l}
n \\
m
\end{array}\right) b_{m} .
\end{aligned}
$$

5. The cases of Fourier transforms. The particular cases $\alpha= \pm_{2}^{1}$ of Theorem 2 are equivalent to the cases of Fourier sine and cosine transforms, respectively, and the Laguerre polynomials can then be expressed in terms of Hermite polynomials $((5), 5 \cdot 6 \cdot 1)$. If we put

$$
F(x)=x^{\natural} f\left(\frac{1}{2} x^{2}\right), \quad G(x)=x^{\frac{1}{2}} g\left(\frac{1}{2} x^{2}\right),
$$

Theorem 2 becomes :

ThEOREM 3. If $F(x)$ belongs to $L^{2}(0, \infty)$, and either $(i) G(x)$ is its cosine transform and $k=0$, or (ii) $G(x)$ is its sine transform and $k=1$, and $F(x)$ and $G(x)$ have the formal expansions

in the sense that

$$
F(x) \sim \sum_{n=0}^{\infty} A_{n} H_{2 n+k}(x / \sqrt{2}), \quad G(x) \sim \sum_{n=0}^{\infty} B_{n} H_{2 n+k}(x / \sqrt{2}),
$$

$$
\begin{aligned}
& A_{n}=\frac{2^{\frac{1}{2}-2 n-k}}{(2 n+k) !} \int_{0}^{\infty} e^{-\frac{1}{2} x^{2} H_{2 n+k}(x / \sqrt{2}) F(x) d x,} \\
& B_{n}=\frac{2^{\frac{1}{2}-2 n-k}}{(2 n+k) !} \int_{0}^{\infty} e^{-\frac{1}{2} x^{2} H_{2 n+k}(x / \sqrt{2}) G(x) d x,}
\end{aligned}
$$


then

$$
\begin{aligned}
& B_{n}=(-1)^{n} \sum_{m=0}^{n} \frac{2^{2 m-2 n}}{(n-m) !} A_{m}, \\
& A_{n}=(-1)^{n} \sum_{m=0}^{n} \frac{2^{2 m-2 n}}{(n-m) !} B_{m} .
\end{aligned}
$$

6. An example. If we put $a_{n}=\left(\frac{1}{2}+\frac{1}{2} a\right)^{n}$ in (8), then ((5) $\left.5 \cdot 1 \cdot 9\right)$

$$
\begin{aligned}
f(x) & =x^{1 \alpha \alpha} \sum_{n=0}^{\infty}\left(1,+!_{2}^{1} a\right)^{n} L_{n}^{(\alpha)}(x) \\
& =x^{j \alpha}\left(!_{2}^{1}-\frac{1}{2} a\right)^{-\alpha-1} \exp \left(-x \frac{1+a}{1-a}\right),
\end{aligned}
$$

for $-3<a<1$. Further, (12) gives

Hence

$$
b_{n}=\sum_{m=0}^{n}(-1)^{m}\left(\begin{array}{l}
n \\
m
\end{array}\right)\left(\begin{array}{l}
1 \\
2
\end{array}+\frac{1}{2} a\right)^{m}=\left(\begin{array}{c}
1 \\
2
\end{array}-\frac{1}{2} a\right)^{n} .
$$

$$
g(x)=x^{\frac{1}{2} \alpha}\left(\frac{1}{2}+\frac{1}{2} a\right)^{-\alpha-1} \exp \left(-x \frac{1-a}{1+a}\right) .
$$

Also, it follows from an extension of Weber's integral (8) that

in accordance with Theorem 2.

$$
g(x)=\int_{0}^{\infty} f(t) J_{\alpha}\left(2 x^{\frac{1}{t}} t^{\frac{1}{2}}\right) d t
$$

7. Fractional transforms. Kober (3) has discussed a class of transformations which can be regarded as fractional powers of the ordinary Hankel transformation. If we re-arrange some of Kober's results so that they pertain to the kernel $J_{\alpha}(2 x)$ instead of the usual Hankel kernel $x J_{\alpha}(x)$ then we obtain :

TheOREM 4. If $f(x)$ belongs to $L^{2}(0, \infty), \alpha$ and $k$ are real, $\alpha>-1$, and

in the sense that

$$
f(x) \sim e^{-x} x^{\dagger \alpha} \sum_{n=0}^{\infty} a_{n} L_{n}^{(\alpha)}(2 x)
$$

$$
a_{n}=\frac{n !}{2^{\alpha+1} \Gamma(n+\alpha+1)} \int_{0}^{\infty} e^{-t t^{1 \alpha}} L_{n}^{(\alpha)}(2 t) f(t) d t,
$$

then there exists a family of transformations $T_{k}$ with the following properties :

(i) $T_{k} f(x) \sim e^{-x} x^{\dagger \alpha} \sum_{n=0}^{\infty} a_{n} e^{2 \pi i n k} L_{n}^{(\alpha)}(2 x)$,

(ii) $T_{k} T_{\imath} f(x)=T_{k+l} f(x), \quad T_{k+1} f(x)=T_{k} f(x)$,

(iii) $T_{1} f(x)=\lim _{T \rightarrow \infty} \int_{0}^{T} f(t) J_{\alpha}\left(2 x^{\left.\frac{1}{t} t^{\mathrm{t}}\right)} d t\right.$,

(iv) If $k$ is not an integer and

then

$$
c_{k}=|\operatorname{cosec} \pi k| \exp \pi i(1+\alpha)\left(\frac{1}{2}-k+[k]\right) \text {, }
$$

$$
T_{k} f(x)=c_{k} \lim _{T \rightarrow \infty} \int_{0}^{T} f(t) J_{\alpha}\left(2 x t^{3}|\operatorname{cosec} \pi k|\right) e^{i(x+t) \cot \pi k} d t
$$


In the present scheme these fractional transforms correspond to the evaluation $I^{k}$ of the $k$ th power of the unit infinite matrix which is given by the diagonal matrix (4).

Now the fractional transformation of the sequence $\left\{a_{n}\right\}$ corresponding by Theorem 1 to the $k$ th power of the transformation (12) is

$$
b_{n}^{\prime}=\sum_{m=0}^{n} e^{2 \pi i m k}\left(\frac{1}{2}-\frac{1}{2} e^{2 n i k}\right)^{n-m}\left(\begin{array}{l}
n \\
m
\end{array}\right) a_{m} .
$$

Hence we can derive a transformation $T_{k}^{\prime}$ which is also a $k$ th power of the transformation with respect to the kernel $J_{\alpha}(2 x)$ if we replace the $b_{n}$ in Theorem 2 by the $b_{n}^{\prime}$ given by (15). In particular, if $f(x)$ is the function (13) considered in $\S 6$, then (15) gives

Hence, in this case,

$$
\begin{aligned}
b_{n}^{\prime} & =\sum_{m=0}^{n} e^{2 \pi i m k}\left(\frac{1}{2}-\frac{1}{2} e^{2 \pi i k}\right)^{n-m}\left(\begin{array}{c}
n \\
m
\end{array}\right)\left(\frac{1}{2}+\frac{1}{2} a\right)^{m} \\
& =\left(\frac{1}{2}+\frac{1}{2} a e^{2 \pi i k}\right)^{n} .
\end{aligned}
$$

$$
\begin{aligned}
T_{k i}^{\prime} f(x) & =x^{\frac{1}{1 \alpha}} \sum_{n=0}^{\infty} b_{n}^{\prime} L_{n}^{\langle\alpha)}(x) \\
& =\left(\frac{1}{2}-\frac{1}{2} a e^{2 \pi i k}\right)^{-\alpha-1} x^{j \alpha} \exp \left(-x \frac{1+a e^{2 \pi i k}}{1-a e^{2 \pi i k}}\right)
\end{aligned}
$$

Thus as $k$ varies (16) gives functions which are $k$ th power transforms of the function (13) with respect to the kernel $J_{\alpha}(2 x)$. We can use this family of fractional transforms to obtain fractional transforms of more general functions by the following device.

Definition 2. If $f(x)$ and $g(x)$ belong to $L^{2}(0, \infty)$, and $F_{a}(x), G_{a}(x)$ are sets of functions belonging to $L^{2}(0, \infty)$ and defined for some set of values of $a$, then we say that $f(x)$ is related to $g(x)$ as the $F_{a}(x)$ are related to the $G_{a}(x)$ if

$$
\int_{0}^{\infty} f(x) G_{a}(x) d x=\int_{0}^{\infty} g(x) F_{a}^{\prime}(x) d x
$$

for all a of the set.

With this terminology we can prove :

THEOREM 5. If $f(x)$ is related to $g(x)$ as the functions (16) are related to the functions (13) for $|a|<1$, and

and

$$
f(x) \sim x^{\mathbf{1} \alpha} \sum_{n=0}^{\infty} a_{n} L_{n}^{(\alpha)}(x)
$$

$$
g(x) \sim x^{j \alpha} \sum_{n=0}^{\infty} b_{n}^{\prime} L_{n}^{(\alpha)}(x),
$$

then the sequences $\left\{a_{n}\right\}$ and $\left\{b_{n}^{\prime}\right\}$ satisfy (15).

Proof. By the assumption of the theorem we have

$$
\begin{aligned}
&\left(1-a e^{2 \pi i k}\right)^{-\alpha-1} \int_{0}^{\infty} f(x) x^{1 \alpha} \exp \left(-x \frac{1+a e^{2 \pi i k}}{1+a e^{2 \pi i k}}\right) d x \\
&=(1-a)^{-\alpha-1} \int_{0}^{\infty} g(x) x^{1 \alpha} \exp \left(-x \frac{1+a}{1-a}\right) d x
\end{aligned}
$$

for $|a|<1$. 
Now for $|2 a /(1+a)|<1$ the right-hand side is equal, by the expansion (5), $5 \cdot 1 \cdot 9$, to

$$
\begin{aligned}
(1+a)^{-\alpha-1} \int_{0}^{\infty} & g(x) e^{-x} x^{\frac{1}{2} \alpha}\left\{\sum_{n=0}^{\infty} L_{n}^{(\alpha)}(x)\left(\frac{2 a}{1+a}\right)^{n}\right\} d x \\
& =(1+a)^{-\alpha-1} \sum_{n=0}^{\infty}\left(\frac{2 a}{1+a}\right)^{n} \int_{0}^{\infty} g(x) e^{-x} x^{\frac{1}{2} \alpha} L_{n}^{(\alpha)}(x) d x \\
& =(1+a)^{-\alpha-1} \sum_{n=0}^{\infty}\left(\frac{2 a}{1+a}\right)^{n} \frac{\Gamma(n+\alpha+1)}{n !} b_{n}^{\prime} .
\end{aligned}
$$

The term by term integration of the series is justified by absolute convergence since

$$
\begin{aligned}
\left|b_{n}^{\prime}\right| & =\frac{n !}{\Gamma(n+\alpha+1)}\left|\int_{0}^{\infty} g(x) x^{\frac{1}{\alpha} \alpha} e^{-x} L_{n}^{(\alpha)}(x) d x\right| \\
& \leqslant \frac{n !}{\Gamma(n+\alpha+1)}\left[\int_{0}^{\infty}|g(x)|^{2} e^{-x} d x\right]^{\frac{1}{2}}\left[\int_{0}^{\infty} e^{-x} x^{\alpha}\left\{L_{n}^{(\alpha)}(x)\right\}^{2} d x\right]^{!} \\
& =\left[\frac{n !}{\Gamma(n+\alpha+1)}\right]^{1}\left[\int_{0}^{\infty}|g(x)|^{2} e^{-x} d x\right]^{\frac{1}{3}} \\
& =O\left(n^{-\frac{1}{2} \alpha}\right) .
\end{aligned}
$$

Hence the series (18) converges absolutely for $|2 a /(1+a)|<1$.

Similarly the left-hand side of (17) is equal to

If we now put

$$
\left(1+a e^{2 \pi i k}\right)^{-\alpha-1} \sum_{n=0}^{\infty}\left(\frac{2 a e^{2 \pi i k}}{1+a e^{2 \pi i k}}\right)^{n} \frac{\Gamma(n+\alpha+1)}{n !} a_{n} .
$$

$$
b_{n}^{\prime}=\sum_{m=0}^{n} e^{2 \pi i m k}\left(1-\frac{1}{2} e^{2 \pi i k}\right)^{n-m}\left(\begin{array}{l}
n \\
m
\end{array}\right) a_{m}^{\prime}
$$

then (18) becomes

$$
\begin{aligned}
(1+a)^{-\alpha-1} & \sum_{n=0}^{\infty}\left(\frac{2 a}{1+a}\right)^{n} \frac{\Gamma(n+\alpha+1)}{n !} \sum_{m=0}^{n} e^{2 \pi i m k}\left(\frac{1}{2}-\frac{1}{2} e^{2 \pi i k}\right)^{n-m}\left(\begin{array}{l}
n \\
m
\end{array}\right) a_{m}^{\prime} \\
= & (1+a)^{-\alpha-1} \sum_{m=0}^{\infty} \frac{a_{m}^{\prime}}{m !} \Gamma(m+\alpha+1)\left(\frac{2 a e^{2 \pi i k}}{1+a}\right)^{m} \sum_{n=m}^{\infty}\left(\begin{array}{l}
n+\alpha \\
n-m
\end{array}\right)\left(\frac{a-a e^{2 \pi i k}}{1+a}\right)^{n-m} \\
= & \left(1+a e^{2 \pi i k}\right)^{-\alpha-1} \sum^{\infty} \frac{a_{m}^{\prime}}{m !} \Gamma(m+\alpha+1)\left(\frac{2 a e^{2 \pi i k}}{1+a e^{2 \pi i k}}\right)^{m} .
\end{aligned}
$$

Equating this to (19) we have

$$
\sum_{n=0}^{\infty}\left(\frac{2 a e^{2 \pi i k}}{1+a e^{2 \pi i k}}\right)^{n} \frac{\Gamma(n+\alpha+1)}{n !}\left(a_{n}-a_{n}^{\prime}\right)=0,
$$

for all relevant $a$. Hence $a_{n}^{\prime}=a_{n}$ and (20) becomes the required result (15).

8. Identity with Kober's transforms. The identity of the fractional transforms of Theorems 4 and 5 is proved if we show that $T_{k} f(x)=T_{k}^{\prime} f(x)$ for the function (13). In this case we have in Theorem 4

$$
\begin{aligned}
a_{n} & =\frac{n !}{2^{\alpha+1} \Gamma(n+\alpha+1)}\left(\frac{1}{2}-\frac{1}{2} a\right)^{-\alpha-1} \int_{0}^{\infty} e^{-x} x^{1 \alpha} L_{n}^{(\alpha)}(2 x) x^{1 \alpha} \exp \left(-x \frac{1+a}{1-a}\right) d x \\
& =\frac{n !}{2^{\alpha+1} \Gamma(n+\alpha+1)}(1-a)^{-\alpha-1} \int_{0}^{\infty} x^{\alpha} L_{n}^{(\alpha)}(x) \exp \left(-\frac{x}{1-a}\right) d x \\
& =2^{-\alpha-1} a^{n}
\end{aligned}
$$


on evaluating this integral ((5), p. 370, problem 19). Hence (i) of Theorem 4 gives

as required.

$$
\begin{aligned}
T_{k} f(x) & =2^{-\alpha-1} e^{-x} x^{i \alpha} \sum_{n=0}^{\infty}\left(a e^{2 \pi i k}\right)^{n} L_{n}^{(\alpha)}(2 x) \\
& =\left(\frac{1}{2}-\frac{1}{2} a e^{2 \pi i k}\right)^{-\alpha-1} x^{\alpha} \exp \left(-x \frac{1+a e^{2 \pi i k}}{1-a e^{2 \pi i k}}\right) \\
& =T_{k}^{\prime} f(x),
\end{aligned}
$$

\section{REFERENCES}

(1) P. Barrucand, Sur les suites réciproques, Comptes Rendus, 230 (1950), 1727-8.

(2) R. G. Cooke, Infinite matrices and sequence spaces (London, 1950), 20.

(3) H. Kober, Wurzeln aus der Hankel-, Fourier-, und aus anderen stetigen Trans. formationen, Quart. J. Math. (Oxford), (1) 10 (1939), 45-59.

(4) M. Plancherel, Contribution à l'étude de la représentation d'une fonction arbitraire par des intégrales définies, Rend. di Palermo, 30 (1910), 289-335.

(5) G. Szegö, Orthogonal polynomials, American Math. Soc. Colloquium Publications, Vol. XXIII (1939).

(6) E. C. Titchmarsh, Hankel transforms, Proc. Cambridge Phil. Soc., 21 (1923), 463-73.

(7) E. C. Titchmarsh, Introduction to the theory of Fourier integrals (2nd edition, Oxford, 1948), 76-83.

(8) G. N. Watson, A treatise on the theory of Bessel functions (2nd edition, Cambridge, 1941). 394 (4).

(9) N. Wiener, The Fourier integral (Cambridge, 1922), Chapter I.

University of New England

ArMmidale, N.S.W.

Australta 